\title{
COMPORTAMIENTO DE LA TEMPERATURA DEL AIRE SUPERFICIAL EN EL GLACIAR FLEMING, PENÍNSULA ANTARTICA, DERIVADO DE UN SENSOR IN SITU
}

\author{
NEAR-SURFACE AIR TEMPERATURE BEHAVIOR ON THE FLEMING GLACIER, ANTARCTIC \\ PENINSULA, DERIVED FROM AN IN-SITU SENSOR
}

Marcelo Guzmán ${ }^{1} \&$ Jorge F. Carrasco ${ }^{1}$

\begin{abstract}
An analysis of the air temperature behavior in the interior of Fleming Glacier (FG), located in Wordie Bay in the central west part of the Antarctic Peninsula, is presented in this study. For this, data obtained in situ by an automatic weather station (AWS) that continuously recorded (every hour) between December 2007 and October 2008, were used. The AWS was installed around 50 $\mathrm{km}$ from the glacier front and at $233 \mathrm{~km}$ to the southeast of Rothera station, at 1,057 m.a.s.l. These data were complemented with meteorological observations registered by AWSs nearby that operate in the peninsula. We found statistically significant correlations among AWSs deployed in FG and the surrounding ones. The mean air temperature at GF AWS range between $-6.3 \pm 0.1$ y $-22.3 \pm 0.3{ }^{\circ} \mathrm{C}$ for the warmest month (January) and the coldest month (August), respectively. While the annual mean, the average between summer (December-January-February) and winter (June-July-August) 2008, was $-12.6{ }^{\circ} \mathrm{C}$. The Zero Isotherm Altitude (ZIA) for those days with air temperature above $0{ }^{\circ} \mathrm{C}$ during the summer at Rothera station was 390 m.a.s.l. The lineal tendency reveals a statistically significant positive trend of $\sim 40 \mathrm{~m}$ for the $1978-2010$ period. The $390 \mathrm{~m}$ height is found around $15-17 \mathrm{~km}$ up slope from the glacier front, revealing that the vast part of the FG and surrounding upper region are below the frozen temperature year-round
\end{abstract}

Key words: Air temperature, automatic weather station, zero isotherm altitude, Fleming glacier.

\section{RESUMEN}

Un análisis sobre el comportamiento de la temperatura del aire al interior del Glaciar Fleming (GF), ubicado en la Bahía Wordie en la parte central oeste de la Península Antártica, es presentado en este 1 Programa de Magister en Ciencias Antárticas, Universidad de Magallanes, Punta Arenas- Chile. marceluma@hotmail.com 
estudio. Para tal efecto se utilizaron datos obtenidos in situ por una estación meteorológica automática (EMA), que registró los datos bajo condiciones de operación continua (cada una hora), entre diciembre de 2007 y octubre de 2008. La EMA fue localiza a $50 \mathrm{~km}$ del frente del glaciar, aproximadamente $233 \mathrm{~km}$ al sur-suroeste de la estación Rothera, y a una altitud de 1.057 m.s.n.m. Estos datos fueron complementados con observaciones meteorológicas registradas por EMAs circundantes que operan en la península. Se encontraron significativas correlaciones estadísticas entre la EMA emplazada en el GF y las EMAs vecinas. La temperatura media mensual del aire registrada en la EMA al interior del GF varió entre $-6,3 \pm 0,1$ y $-22,3 \pm 0,3^{\circ} \mathrm{C}$ para el mes más cálido (enero) y frío (agosto), respectivamente. Mientras que el promedio anual, calculado con la media de verano e invierno de fue de $-12.6^{\circ} \mathrm{C}$ para el año 2008 . La altitud media de la isoterma Cero (ZIA, Zero Isotherm Altitud) para los días con temperaturas sobre $0{ }^{\circ} \mathrm{C}$, durante el verano (diciembre, enero y febrero) en la estación Rothera, alcanzó los 390 m.s.n.m. La tendencia lineal para la ZIA revela un incremento en altura estadísticamente significativo de 40 $\mathrm{m}$ para el periodo 1978-2010. La cota $390 \mathrm{~m}$ es alcanzada a alrededor de $15-17 \mathrm{~km}$ al interior del frente del glaciar, revelando que la mayor parte del GF y sus alrededores están bajo las temperaturas de congelamiento durante todo el año.

Palabras clave: temperatura del aire, estaciones meteorológicas automáticas, altura de la isoterma cero, Glaciar Fleming.

\section{INTRODUCCIÓN}

La Península Antártica (PA) es una cordillera con varias cimas que sobrepasan los 2000 metros de altitud, extendiéndose por alrededor de 1300 $\mathrm{km}$ hacia el norte, desde la base del continente (Tierra de Ellsworth) hasta el Paso de Drake. Cerca de dos tercios de la PA se ubican al norte de los $70^{\circ} \mathrm{S}$ separando el océano Pacifico del Atlántico, y su dirección norte-sur es casi perpendicular a los vientos los oestes (westerlies) que predominan en la zona. A ambos lados de la península, existe un gran número de glaciares que llegan hasta los márgenes continentales en la costa drenando, ya sea, en las plataformas de hielo adyacentes, o directamente en el mar.

Las empinadas montañas y la circulación atmosférica que predomina en la región determinan dos ambientes climáticos distintos a cada lado de la PA. En el lado del Mar de Bellingshausen encontramos un clima sub polar con temperaturas medias anuales de alrededor de -3.3 a $-5,6^{\circ} \mathrm{C}$; mientras que en el lado del Mar de Weddell, el flujo predominante del sur trae aire frío desde el interior del continente definiendo un clima polar continental con temperaturas medias anuales cercanas a los -9 a $-12^{\circ} \mathrm{C}$, (Schwerdtfeger, 1984; Reynolds, 1981). Smith y Stammerjohn (1996) indican una diferencia de la temperatura del aire de aproximadamente $7^{\circ} \mathrm{C}$ entre la costa este y oeste de la PA, en sitios de latitud y altitud comparables. Este contraste climático también se manifiesta en otros parámetros como la nubosidad, humedad relativa y la precipitación/ acumulación los que son mayores en el lado del Mar de Bellingshausen que en el lado del Mar de Wedell (Vaughan et al. 1999).

Desde medidos del 2007 a fines del 2008, un proyecto Chileno enmarcado en el Año Polar Internacional (IPY; International Polar Year) fue realizado por el Centro de Estudios Científicos (CECS) en colaboración con la Dirección Meteorológica de Chile, y el apoyo logístico del Instituto Chileno Antártico (INACH) y la Fuerza Aérea Chilena (FACH). El objetivo del proyecto fue mejorar la comprensión de la actual dinámica del Glaciar Fleming (GF de aquí en adelante), localizado alrededor de los $69,5^{\circ} \mathrm{S}, 66^{\circ} \mathrm{W}$ con una extensión de alrededor 45 km (Fig. 1), en una región donde actualmente se están desarrollando cambios medioambientales significativos; los que han quedado en evidencia, por ejemplo, por el retroceso y colapso de la plataforma de hielo Wordie (WIS de aquí en adelante por su siga en inglés). Una estación meteorológica automática (EMA) fue instalada al interior del GF para el estudio de las variables atmosféricas. Este artículo describe el comportamiento de la temperatura superficial del aire registrada in situ, en un lugar con escasas observaciones y registros reales. Las otras variables 
meteorológicas serán descritas en sucesivos artículos, pero pueden verse preliminarmente en Guzmán (2014) y los aspectos glaciológicos del proyecto están descritos en Wendt et al. (2010). La siguiente sección resume antecedentes relevantes y de interés para introducir al lector no familiarizado con el conocimiento físico de la PA, luego se describe la EMA y la metodología de procesamiento de los datos. Resultados del análisis del comportamiento de la temperatura del aire y las conclusiones se describen en la penúltima y última sección, respectivamente.

\section{ANTECEDENTES PRELIMINARES}

\section{El calentamiento en la Península Antártica}

Con el objeto de investigar el cambio climático que ha experimentado el continente antártico, diversos estudios han utilizado los registros meteorológicos de las estaciones de investigación emplazadas en el continente, algunos de los cuales se remontan al Año Geofísico Internacional, 1957. Estos estudios muestran que el lado oeste de la PA ha experimentado el mayor calentamiento superficial anual del planeta durante los últimos 50 años (Vaughan et al. 2001; 2003; Turner et al. 2006; Marshall et al. 2006; Din et al. 2011; Carrasco, 2013), alcanzando un aumento máximo de 0.55 ${ }^{\circ} \mathrm{C} /$ década en la estación Faraday/Vernadsky (Turner et al. 2006). Según Vaughan (2006), los registros de la estación Faraday/Vernadsky para el periodo 1950 - 2000, muestran un incremento de un $74 \%$ en el número de días con temperatura $>$ $0{ }^{\circ} \mathrm{C}$ (días grado positivo o Positive Degree-Days, PDDs; también denominados días cálidos o warm days, wd). Además, los datos de las estaciones en la zona oeste de la península han demostrado que los cambios de la temperatura media anual, son forzados principalmente por el incremento de la temperatura mínima y estacionalmente por la temperatura invernal (Carrasco, 2013).

La magnitud del calentamiento antártico invernal y su incidencia en el incremento de la temperatura en la troposfera antártica fue ya indicado por Turner et al. (2006), concluyendo que en la atmósfera antártica, el máximo calentamiento se produce en la tropósfera media (400 a 600 $\mathrm{hPa}$ ). De esta forma, la variación de la temperatura invernal para el periodo 1971-2003, fue de 0.15 ${ }^{\circ} \mathrm{C} /$ década a nivel superficial y de $0.7^{\circ} \mathrm{C} /$ década en la tropósfera media; mientras que se registra un enfriamiento de $-0.1^{\circ} \mathrm{C} /$ década en la estratósfera (entre los 200 y $50 \mathrm{hPa}$, con el registro máximo en los $100 \mathrm{hPa}$ ). Particularmente en la PA, Carrasco (2012) encontró un aumento de $0.35^{\circ} \mathrm{C} /$ década en tropósfera baja y media de los niveles de 850 y $500 \mathrm{hPa}$ y un leve enfriamiento de 0.17 ${ }^{\circ} \mathrm{C} /$ década en la estratósfera del nivel $100 \mathrm{hPa}$, para el período 1955-1998 en las estaciones de radiosonda de Bellingshausen y Rothera.

\section{El impacto en la criósfera}

Los efectos del calentamiento regional en la criósfera de la PA pueden ser variados, como una disminución en la extensión del hielo marino, un retroceso sostenido de las plataformas de hielo, y una aceleración y pérdida de masa de los glaciares. Al respecto, Hock et al. (2009) señala que la pérdida de masa superficial (sin considerar las pérdidas de masa por calving) de todos los glaciares de montaña y de las capas de hielo de la Antártica han contribuido en $0.22 \pm 0.16 \mathrm{~mm} \mathrm{a}^{-1} \mathrm{al}$ aumento global del nivel del mar. Todo este aporte proviene principalmente de la PA y corresponde a un $28 \%$ del total global estimado. Esto se debe principalmente al excepcional calentamiento que ha experimentado la PA y a su alta sensibilidad ante el incremento de la temperatura; similar a la situación de Islandia, la Patagonia y Alaska. A modo general, Hock et al. (2009) señala que los glaciares en ambientes marítimos son más sensibles a los incrementos en temperatura que aquellos glaciares continentales.

El conocimiento y entendimiento de la interacción entre las plataformas de hielo y los glaciares y la dinámica de dicha relación es de suma importancia para poder prever, ante distintos escenarios de cambio climático, el incremento de la descarga líquida, o agua de derretimiento desde los glaciares interiores hacia los océanos, aportando de esta manera al incremento en el aumento del nivel global del mar. En la región de la PA el derretimiento se produce a una temperatura superficial umbral de $-2.5^{\circ} \mathrm{C}$ (promedio mensual); a su vez, el área donde se produce derretimiento superficial aumenta rápidamente con la temperatura, estimándose un 
incremento en la superficie de $3.5 \times 10^{6} \mathrm{~d} \mathrm{~km}^{2}$ por cada grado en que se incrementa la temperatura en verano (Zwally \& Fiegles, 1994). Por otro lado, el límite espacial para la existencia y mantención de las plataformas de hielo, límite de viabilidad, aparentemente corresponde con la ubicación de la isoterma $0^{\circ} \mathrm{C}$ en verano (Vaughan \& Doake, 1996), la cual se ha desplazado hacia el sur producto del calentamiento atmosférico que ha experimentado la región de la PA.

Los registros recientes de periodos de retroceso y posteriores eventos de colapso de plataformas de hielo, permiten reconocer un patrón de desplazamiento hacia el sur de dicho límite climático. Según los datos disponibles y la distribución de las plataformas de hielo, la ubicación de la isoterma $0^{\circ} \mathrm{C}$ en enero coincide con la ubicación de la isoterma media anual $-5^{\circ} \mathrm{C}$ (mapeada por Reynolds, 1981). Morris \& Vaughan (2003) indicaron que una plataforma de hielo se torna inviable, cuando sufre una serie de retrocesos progresivos durante un periodo de varios años, sin registrar avances significativos. Por su parte, muestran resultados que indican que el límite de viabilidad para las plataformas de hielo de la PA esta dado por la isoterma media anual $-9{ }^{\circ} \mathrm{C}$, el aumento observado de la temperatura ha desplazado este límite hacia el sur. Esto concuerda con su estudio, donde muestran que de todas las plataformas analizadas, las cinco ubicadas al sur de la isoterma $-9^{\circ} \mathrm{C}$, no muestran cambios relevantes. Para las plataformas ubicadas al norte de dicho límite, los registros muestran que a contar de 1947 se ha perdido un área total de $28.117 \mathrm{~km}^{2}$ de plataformas de hielo (Cook \& Vaughan, 2010). Analizando el fenómeno a una escala interdecadal, se evidencia una disminución sostenida del área total de las plataformas de hielo en la PA, comenzando en 1970s y prolongándose hasta el presente. De esta forma, el patrón de retroceso sostenido de las plataformas de hielo ubicadas al oeste de la PA, es un indicador del cambio climático local que está afectando dicha zona en épocas recientes.

En respuesta a este cambio climático local ocurrido durante las últimas décadas en la PA, las plataformas de hielo han experimentado un proceso sostenido de retroceso y disminución de sus áreas y varios eventos de colapso muy rápidos (ver Cook \& Vaughan, 2010) quienes presenta un completo resumen). El retroceso y la desintegración por colapso de las plataformas de hielo afectan directamente los glaciares interiores que drenan hacia ellas, produciéndose un incremento en las aceleraciones del flujo de hielo y un adelgazamiento de dichos glaciares. De esta forma, el fenómeno es susceptible de afectar el comportamiento y estabilidad de los glaciares interiores al modificar el balance de masa, mediante adelgazamiento, retroceso y consiguiente pérdida de masa, aumentando la descarga líquida hacía los océanos, favoreciendo la reducción de la salinidad y eventuales cambios en las corrientes marinas, afectando el clima a nivel local, regional $y$ potencialmente a nivel global, $y$ aportando al incremento del nivel del mar.

Como consecuencia de este calentamiento regional, las plataformas de hielo adyacentes a la PA que han colapsado y retrocedido durante las últimas décadas son las plataformas de Muller, Jones, George VI, Wordie, Larsen A y Larsen B (Vaughan \& Doake, 1996 entre muchos otros). La rápida desintegración de la plataforma WIS, así como los cambios en el comportamiento de los glaciares en el lado oeste de la península, son evidencias de la tendencia al calentamiento en esta región (Smith \& Stammerjohn, 1996). Uno de los eventos más recientes tuvo lugar en la plataforma de hielo de Wilkins, la cual experimentó el colapso del puente de hielo que conectaba la plataforma con la isla Charcot (Cook \& Vaughan, 2010).

El retroceso y colapso de las plataformas de hielo no contribuye directamente de manera sustancial al incremento del nivel del mar, ya que el hielo contenido en ellas flota actualmente en un equilibrio hidrostático con los océanos (Wendt et al. 2010). No obstante lo anterior, el incremento de las aceleraciones en los flujos de hielo de los glaciares interiores como consecuencia del colapso de una plataforma, provoca una mayor descarga de hielo al océano que normalmente no es equilibrada mediante la acumulación de nieve en los sectores altos de los glaciares. La desintegración de las plataformas de hielo puede cambiar la dinámica de los glaciares, que anterior a dicho colapso, drenaban hacia plataformas de hielo sólidas. La remoción del soporte que otorgaban las plataformas de hielo, aumenta la velocidad de los glaciares, drenando directamente al mar y por ende contribuyendo al aumento del 
nivel del mar por el incremento de los flujos de hielo hacia el océano (Rignot et al. 2004).

\section{Plataforma de hielo de Wordie (WIS)}

La WIS ha estado retrocediendo en una serie de eventos desde la década del 50s, y en 1992 sufrió la pérdida de área más dramática experimentada en todas las plataformas de hielo de la PA (Vaughan \& Doake, 1996; Cook \& Vaughan, 2010). La WIS se redujo desde alrededor de $1.917 \mathrm{~km}^{2}$ en 1960 a $139 \mathrm{~km}^{2}$ en 2008/2009, alcanzando una pérdida de $1.218 \mathrm{~km}^{2}$ de hielo (Cook \& Vaughan, 2010). El GF es uno de los mayores tributarios de la WIS y junto a otros glaciares vecinos ya han experimentado cambios debido a la desintegración de la plataforma de hielo (Cook \& Vaughan, 2010; Wendt et al. 2010). Rignot et al. (2005) mostró que en 1996, el GF se estaba moviendo a velocidades $50 \%$ mayores que las mediciones obtenidas en tres posiciones el año 1974 (Doake, 1975), concluyendo que la aceleración se debía al colapso de la WIS a comienzos de los 70`s. La aceleración que está sufriendo el GF fue confirmada por Wend et al. (2010), quienes compararon las mediciones tomadas por Doake en 1974, con datos recolectados con GPS durante los veranos australes de 2007/2008 y 2008/2009, encontrando que las velocidades en los últimos tiempos son un 40 a $50 \%$ más altas.

Una comparación de la topografía superficial del hielo mapeada en 2004 y 2008, utilizando un sistema de escaneo laser aerotransportado, ha revelado un adelgazamiento general del hielo que alcanza un máximo de $4.1 \mathrm{~m} / a n ̃ o$, cerca del frente

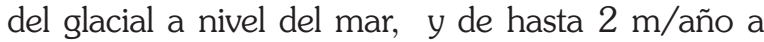
una altitud aproximada de 1.100 m.s.n.m. (Wendt et al. 2010). Junto con lo anterior, se ha señalado una descarga de hielo $84 \%$ mayor a la acumulación de nieve en el glaciar Fleming (Rignot et al. 2005). Todo lo anterior estaría confirmando un balance de masa negativo en el GF.

\section{ESTACIÓN METEOROLÓGICA AUTOMÁTICA}

Un equipo del CECS realizó un total de tres campañas en la parte alta del GF (límite norte del

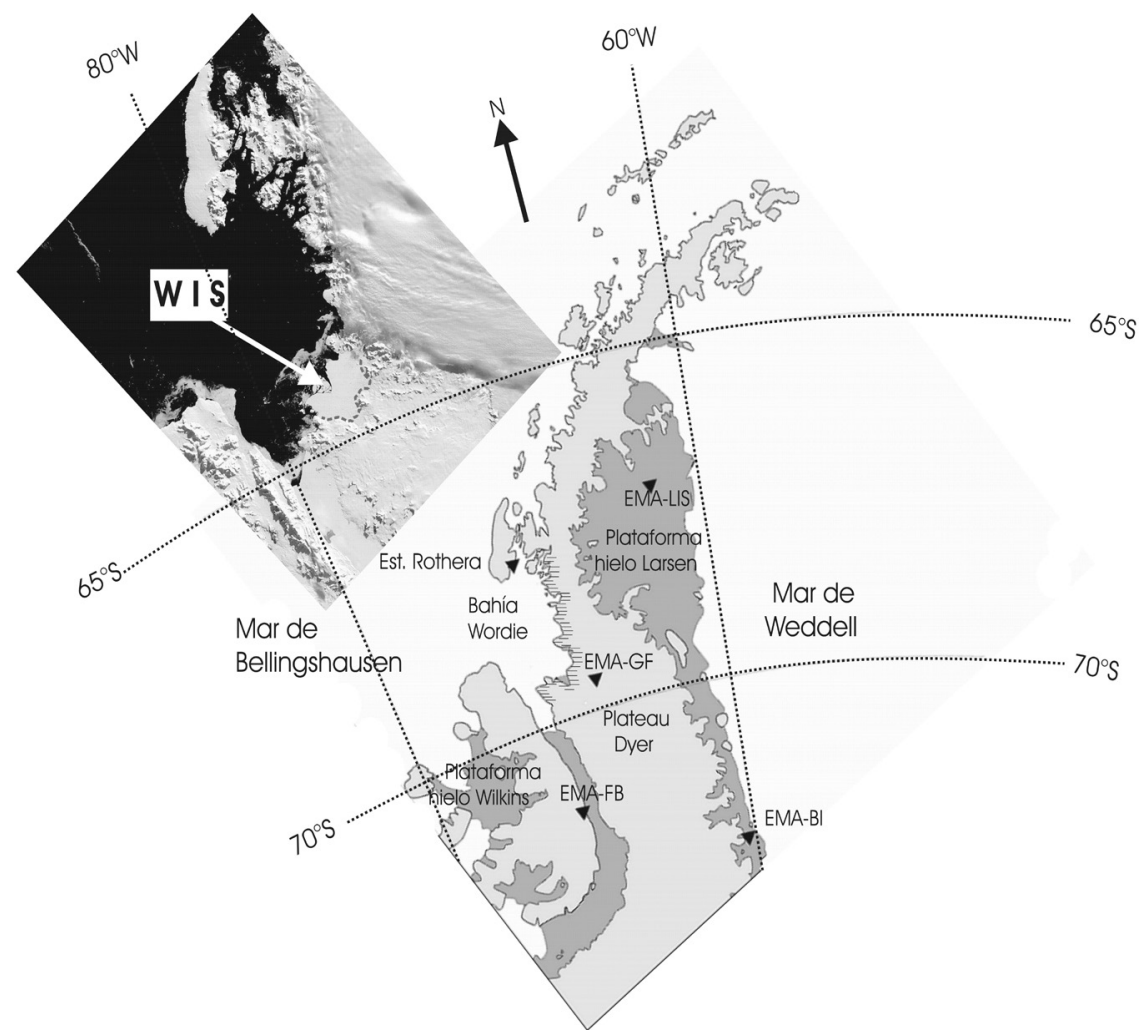

Fig. 1. Emplazamiento de las estaciones meteorológicas consideradas en el presente estudio, cercanas al Glaciar Fleming. 
Tabla 1. Tipo y característica de los sensores meteorológicos de la EMA instalada en el glaciar Fleming.

\begin{tabular}{|c|c|c|c|}
\hline Sensor & Tipo & Rango & Precisión \\
\hline Presión del aire & Vaisala PTB101B & 600 a $1.060 \mathrm{hPa}$ & $4 \mathrm{hPa}$ \\
\hline Temperatura del aire & Vaisala HMP35AC & $-80 \mathrm{a}+56^{\circ} \mathrm{C}$ & $0.3^{\circ} \mathrm{C}$ \\
\hline Temperatura del aire & Campbell Chromel Constant & $-40 \mathrm{a}+40{ }^{\circ} \mathrm{C}$ & $0.1^{\circ} \mathrm{C}$ \\
\hline Humedad relativa & Vaisala HMP35AC & 0 a $100 \%$ & $\begin{array}{l}2 \%(\mathrm{RH}<90 \%) \\
3 \%(\mathrm{RH}>90 \%)\end{array}$ \\
\hline Velocidad del viento & Young 05103 & 0 a $60 \mathrm{~ms}^{-1}$ & $0.3 \mathrm{~m} \mathrm{~s}^{-1}$ \\
\hline Dirección del viento & Young 05103 & 0 a $360^{\circ}$ & $3^{\circ}$ \\
\hline $\begin{array}{l}\text { Pyranometer } \\
\text { shortwave radiation }\end{array}$ & Kipp en Zonen CNR1 & 305 a $2.800 \mathrm{~nm}$ & $2 \%$ \\
\hline $\begin{array}{l}\text { Pyrradiometer net short- and } \\
\text { longwave radiation }\end{array}$ & Kipp en Zonen CNR1 & 5.000 a 50.000 & $15 \mathrm{~W} \mathrm{~m}^{-2}$ \\
\hline Altura de nieve & Campbell SR50 & 0.5 a $10 \mathrm{~m}$ & $0.01 \mathrm{~m}$ ó $0.4 \%$ \\
\hline
\end{tabular}

Plateau Dyer, Fig. 1), en noviembre/diciembre del 2007, 2008 y 2009. Durante la primera campaña se instaló una estación meteorológica automática en el interior del GF (EMA-GF, Estación Meteorológica Automática en Glaciar Fleming), en las coordenadas $69,53^{\circ} \mathrm{S}, 66.01^{\circ} \mathrm{W}$, a 1.057 m.s.n.m. Luego, se realizó una segunda campaña, en diciembre del 2008, permitiendo recolectar los datos grabados durante el primer año de registros. Lamentablemente, durante la tercera campaña, en diciembre del 2009, no se pudo localizar la EMA, probablemente al ser destruida por los fuertes vientos o por haber quedado cubierta totalmente por la nieve y desplazada de su ubicación ya que no fue posible localizarla en las coordenadas GPS registradas originalmente en la campaña de 2008. Por lo tanto, para este estudio se analizaron los datos meteorológicos registrados entre la primera y segunda campaña (2007-2008).

La EMA instalada en el GF era del tipo Campbell científica (Fig. 2), energizada por paneles solares y baterías de $12 \mathrm{~V}$. Los sensores fueron instalados en una torre de $6.1 \mathrm{~m}$ de alto y enterrada $2 \mathrm{~m}$ bajo la superficie de la nieve (Tabla 1).

Los sensores fueron instalados en dos niveles, medidos desde la superficie de nieve: 2.5 y $1.5 \mathrm{~m}$. Esta altura fue elegida dada las restricciones de la torre disponible. La instalación se efectuó en el mes de noviembre

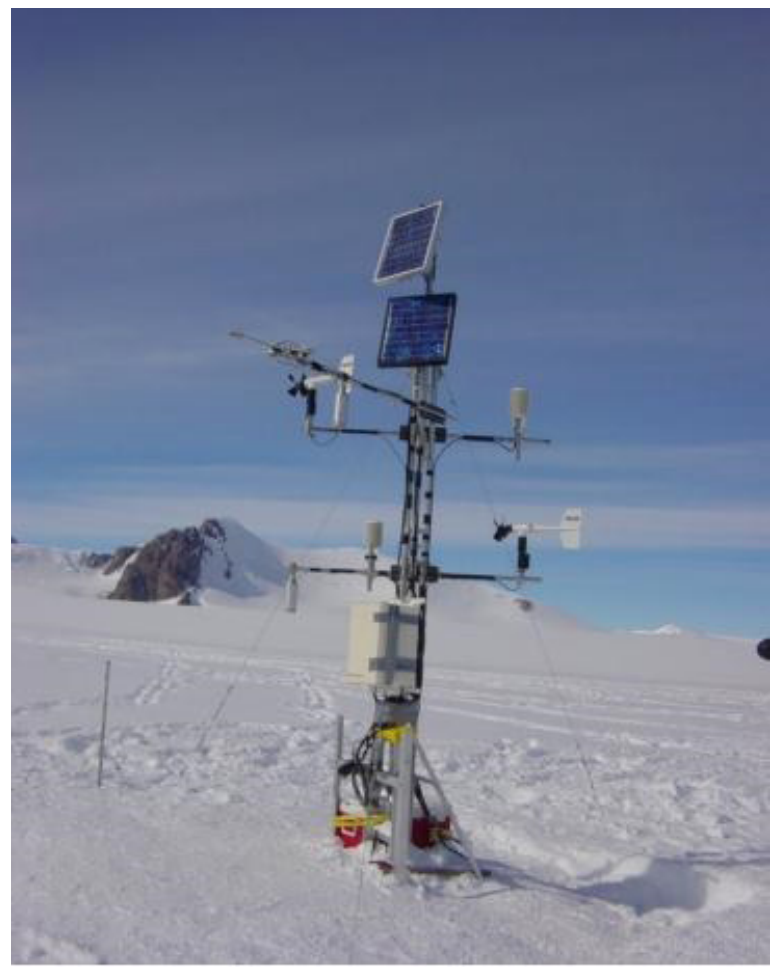

Fig. 2. Fotografía de la EMA instalada en el glaciar Fleming (Fig. 1) en noviembre de 2007 por Jens Wendt (Q.E.P.D.).

de 2007 quedando operativo a partir del 30 de ese mes. Los datos se registraron en un data logger a intervalos de una hora. En diciembre de 2008, durante la segunda campaña, se 
descargaron los datos desde la EMA y se reinstaló la estación para el año siguiente, perdiéndose definitivamente y no siendo encontrada cuando se visitó nuevamente el área el año 2009.

Existen otros datos meteorológicos disponibles, registrados por otras EMAs instaladas en la región (Fig. 1). Se utilizaron datos disponibles de tres estaciones del Programa de la Universidad de Wisconsin (Tabla 2): Fossil Bluff (71 $33^{\circ} \mathrm{S}, 68^{\circ} 28^{\circ} \mathrm{W}$, a 63 m.s.n.m., al oeste de la PA, EMA-FB); Plataforma de hielo de Larsen $\left(67^{\circ} 01^{\circ} \mathrm{S}, 61^{\circ} 55^{\circ} \mathrm{W}\right.$, a 17 m.s.n.m., al este de la PA, EMA-LIS); Isla Buttler (72 $21^{\prime} \mathrm{S}$, $60^{\circ} 17^{\circ} \mathrm{W}$, a 91 m.s.n.m., al este de la PA, EMA-BI). Los datos fueron obtenidos a través de internet en la página http://amrc.ssec.wisc. edu/aws.html. Además, están disponibles los datos diarios y mensuales de la estación Rothera $\left(67^{\circ} 34^{\circ} \mathrm{S}, 68^{\circ} 08^{\circ} \mathrm{W}\right.$, a 32 m.s.n.m., en sector oeste de la PA), que fueron descargados desde el Proyecto READER (http://antarctica.ac.uk/ met/READER/) del Servicio Antártico Británico (British Antarctic Survey). Todos estos datos adicionales fueron utilizados para realizar análisis de comparación con los datos recolectados por la EMA-GF y para caracterizar la meteorología de la región más allá del período de observación. Además, de esta misma fuente se obtuvieron los datos de radiosonda de la estación Rothera. Como se indicó, en este artículo sólo analizamos la serie de temperatura del aire superficial registrado por el sensor superior, el que es comparado con los datos de las estaciones de la región.

Los datos horarios fueron utilizados para obtener el promedio diario y para analizar el comportamiento de las diferentes variables medidas en la EMA-GF. Los datos fueron "suavizados" aplicando un proceso de filtrado exponencial (Rosenblüth et al. 1997):

$y_{t}=c \cdot x_{t}+(1-c) \cdot y_{t-1} \quad t=2,3, \ldots \ldots n$

$z_{t}=c \cdot y_{t}+(1-c) \cdot z \quad t=(n-1),(n-2), \ldots \ldots 1$

donde (1) y (2) son respectivamente, el primer filtrado de la serie de datos hacia adelante y el segundo filtrado, hacia atrás desde el final de la serie resultante del primer filtrado, permitiendo "suavizar" los datos. El primer valor yt es el promedio de los primeros diez (10) valores de la serie y $z_{t}$ corresponde al valor final del primer suavizado hacía adelante. El grado de suavizado (c) puede variar desde 1 (máximo, reproduce los datos originales) a 0 (da como resultado una línea recta). En este caso, se usó $c=0,11$ lo que permite filtrar las frecuencias altas como la variabilidad inter-horarias e inter-diaria pero mantiene el comportamiento de largo plazo.

\section{RESULTADOS}

El promedio de la temperatura del aire registrada en la EMA-GF fue de -12,1 \pm 0,3 (error estándar) para el período de registro de diciembre 2007 a octubre 2008. Para el verano (diciembreenero-febrero) fue $-6,8 \pm 0,1{ }^{\circ} \mathrm{C}$ y en invierno (junio-julio-agosto) $-18,3 \pm 0,1{ }^{\circ} \mathrm{C}$, lo que da un promedio anual de $-12,6^{\circ} \mathrm{C}$. La temperatura media mensual registrada en la EMA-GF varió entre -6,3 $\pm 0,1{ }^{\circ} \mathrm{C}$ para enero (mes más cálido) y -22,3 \pm $0,3{ }^{\circ} \mathrm{C}$ para agosto (mes más frío), con un máximo diario absoluto de la temperatura de $0,7^{\circ} \mathrm{C}$ y un mínimo absoluto de $-41,3^{\circ} \mathrm{C}$. La Figura 3 muestra

Tabla 2. Nombre y ubicación de las EMA utilizadas en este estudio (Fig. 2).

\begin{tabular}{lccc}
\hline \multirow{2}{*}{ Nombre de la estación } & \multicolumn{2}{c}{ Ubicación } & Altitud \\
& Latitud (S) & Longitud (W) & m s.n.m \\
\hline Estación Glaciar Fleming (EMA-FG) & $69^{\circ} 32^{\prime}$ & $66^{\circ} 01^{\prime}$ & 1057 \\
Fossil Bluff (EMA-FB) & $71^{\circ} 33^{\prime}$ & $68^{\circ} 28^{\prime}$ & 63 \\
Larsen Ice Shelf (EMA-LIS) & $67^{\circ} 01^{\prime}$ & $61^{\circ} 55^{\prime}$ & 17 \\
Buttler Island (EMA-BI) & $72^{\circ} 21^{\prime}$ & $60^{\circ} 17^{\prime}$ & 91 \\
Rothera & $67^{\circ} 34^{\prime}$ & $68^{\circ} 08^{\prime}$ & 32 \\
\hline
\end{tabular}


la oscilación media diaria de la temperatura en verano e invierno, la cual fluctúa $4^{\circ}\left(-4,6\right.$ y $\left.-8,6^{\circ} \mathrm{C}\right)$ y $0,9^{\circ}\left(-18,0\right.$ y $\left.-18,9^{\circ} \mathrm{C}\right)$ entre la máxima y mínima temperatura, respectivamente. Durante la estación estival de 2007-2008 en la EMA-GF, sólo 9 días presentaron una temperatura máxima del aire mayor a $0^{\circ} \mathrm{C}$ en al menos una hora de observación, $y$ se registraron 125 horas con temperatura igual o superior a $-2,5{ }^{\circ} \mathrm{C}$ distribuidos en 29 días (con al menos una hora), valor considerado umbral para el derretimiento en la PA (Zwally \& Fiegles, 1994). El promedio diario con temperatura $>-2,5$ fue de 4 horas y el evento de mayor duración fue de 18 horas consecutivas con temperatura mayor a este umbral. Análisis compuesto de los días con temperatura $>-2,5$ muestra una circulación ciclónica centrada al oeste de la PA, en las cercanías del Mar de Amundsen, lo que da origen a un flujo relativamente más cálido afectando el noroeste en la península, mientras que el compuesto para días con temperatura $<-2,5$ la circulación ciclónica se centra en el Mar de Weddell originando un flujo del sur y sureste sobre la península, advectando aire relativamente más frío en la región.

La temperatura promedio del aire registrada por la EMA-GF durante el periodo de observación, fue comparada estadísticamente con los promedios diarios y mensuales de las estaciones meteorológicas vecinas. La Tabla 3 muestra la correlación entre las estaciones meteorológicas utilizadas en este estudio. Las mayores correlaciones se encontraron entre EMA-GF y las dos estaciones occidentales, Rothera y EMA-FB; no obstante lo anterior, las correlaciones también son buenas con las estaciones localizadas en el lado este de la península (EMA-LIS y EMA$\mathrm{BI})$. Estadísticamente, todas las correlaciones son significativas al 95\% de nivel de confianza.

La Fig. 4 muestra el comportamiento de la temperatura del aire alrededor de la EMA-GF
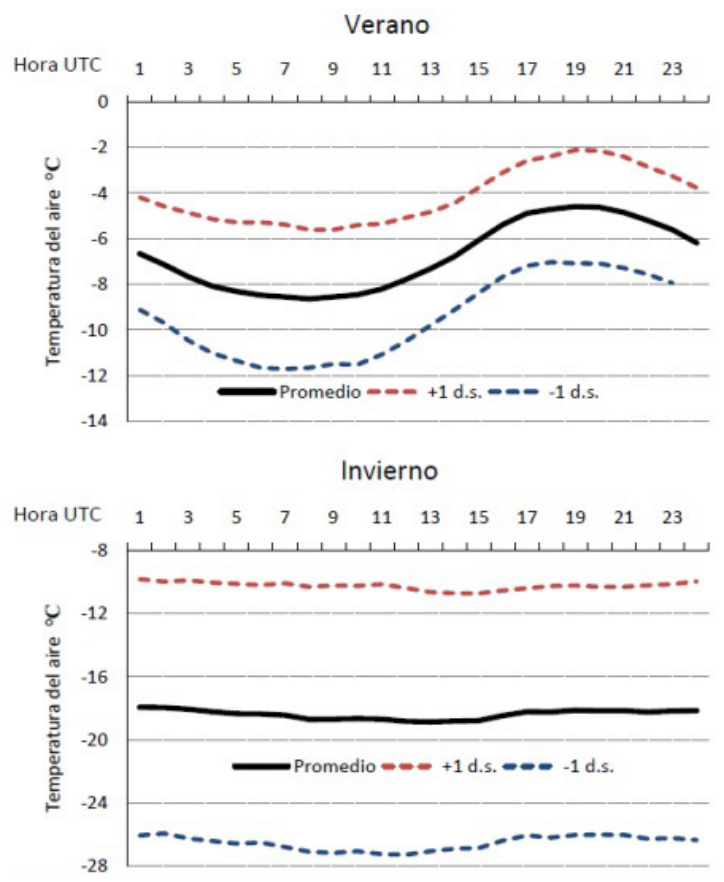

Fig. 3. Promedio de la oscilación diaria de la temperatura del aire para verano e invierno de 2008 , junto con \pm 1 desviación estándar (d.s.), registrada en la estación del GF.

para el periodo registrado, comparándolo con las temperaturas registradas en las estaciones circundantes. La variación inter-diaria fue filtrada utilizando las ecuaciones 1 y 2 , lo que permite ver el comportamiento estacional y sinóptico. El análisis de las series de tiempo y de las correlaciones, muestran un comportamiento similar de la temperatura del aire a ambos lados de la PA; no obstante, un ambiente más frío es registrado en el lado este de la península, en las estaciones EMA-LIS y EMAIB. Las variaciones inter-inter-diarias (eventos de aumento y disminución de la temperatura mayor a un día) son similares en todas las estaciones incluso a ambos las de la península indicando la

Tabla 3. Correlaciones entre la temperatura diaria del aire de la EMA ubicada en el glaciar Fleming y las EMAs del Programa de la Universidad de Wisconsin y la estación de Rothera (Servicio Antártico Británico). Todas las correlaciones son significativas con un 95\% de nivel de confianza.

\begin{tabular}{lllll}
\hline Correlación & EMA-FB & Rothera & EMA-LIS & EMA-BI \\
\hline EMA-FG & 0.85 & 0.92 & 0.72 & 0.53 \\
EMA-FB & & 0.76 & 0.73 & 0.52 \\
Rothera & 0.76 & & 0.64 & 0.42 \\
EMA-LIS & 0.73 & 0.64 & & 0.73 \\
\hline
\end{tabular}




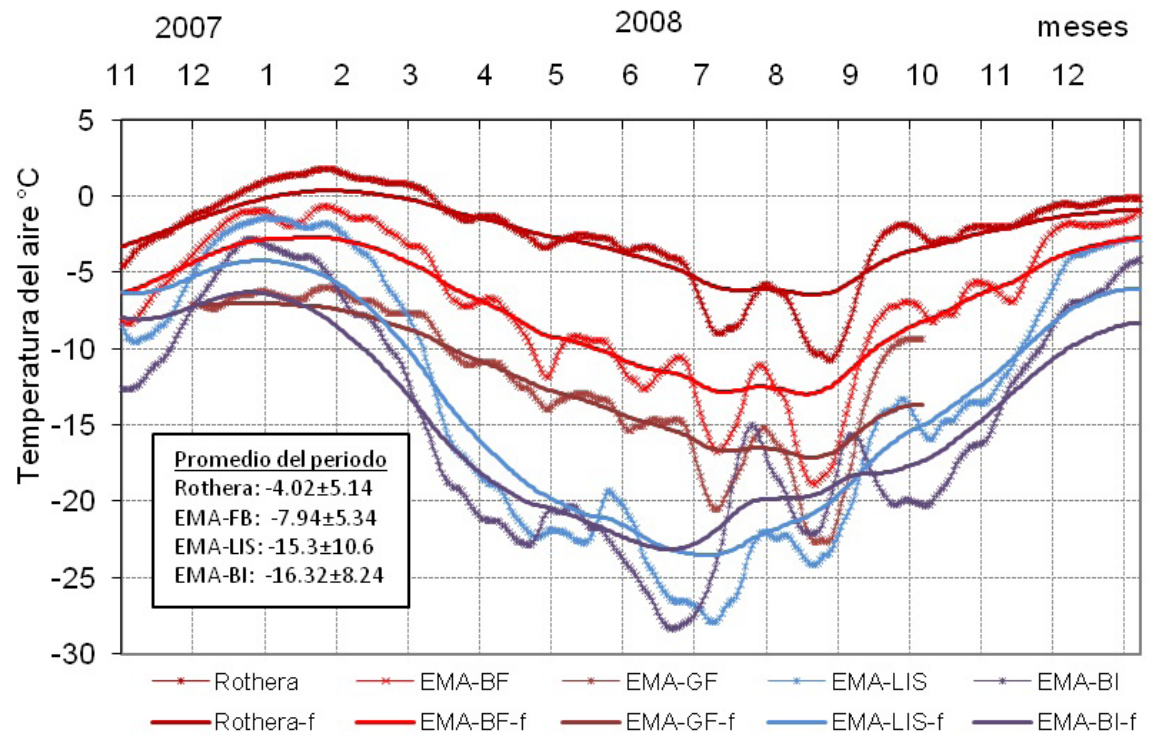

Fig. 4. Comportamiento de la temperatura del aire (superficial) registrada en las estaciones meteorológicas ubicadas en la PA, cercanas al glaciar Fleming, entre noviembre 2007 - diciembre 2008. Se filtraron los datos para permitir ver el comportamiento estacional y sinóptico. Cuadro inserto muestra los valores promedios ( \pm 1 desviación estándar) de la temperatura del aire para el periodo concurrente de observación de las EMAs.

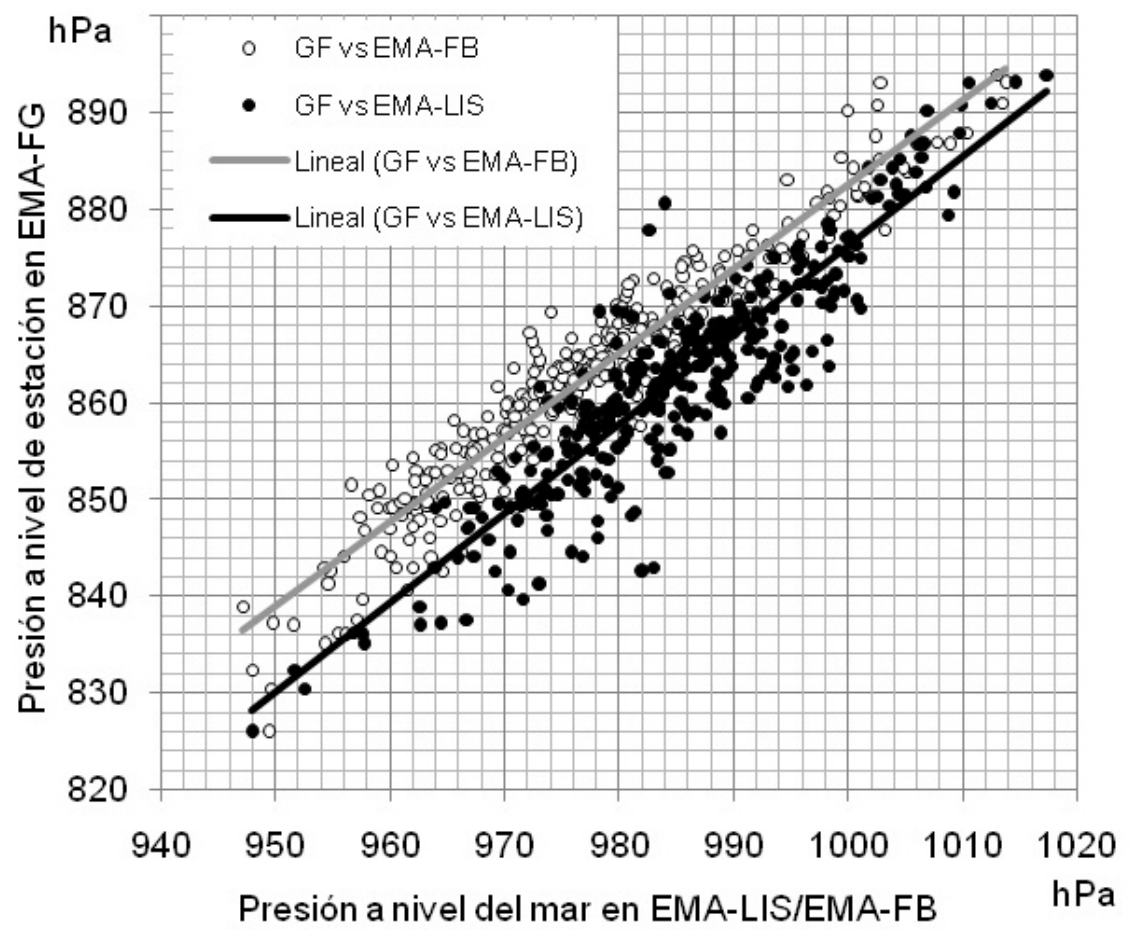

Fig. 5. Correlaciones entre los promedios diarios de presión en las estaciones EMA-GF y EMA-FB (círculos abiertos y línea gris), y entre EMA-GF y EMA-LIS (círculos llenos y línea negra). 
influencia del paso de perturbaciones de escala sinóptica en la región. Esto queda confirmado por el comportamiento de la presión registrado en la EMA-GF y las estaciones vecinas que presentan una alta correlación, estadísticamente significativa. Por ejemplo, la correlación diaria entre EMAGF y EMA-FB, y entre EMA-GF y EMA-LIS es respectivamente de 0,96 y 0,91 (Fig. 5).

Dado que la EMA-GF y la estación de Rothera presentan un comportamiento similar de la temperatura del aire con una alta correlación (0.92) a un $95 \%$ de confianza estadística para el período diciembre 2007 - octubre 2008, se procedió a ajustar la temperatura media mensual de Rothera a la locación de la EMA-GF de acuerdo a la expresión $\mathrm{T}_{\text {EMA-FG }}=1.3114 \times \mathrm{T}_{\text {Rothera }}-8.3952$ (Fig. 6a). Según los datos de la estación Rothera, las medias mensuales del año 2008 estuvieron dentro de \pm 1 variación estándar del del promedio 1978-2010, por lo que se puede considerar que el periodo de registro por la EMA-GF está dentro del rango de un año normal. De esta manera, los datos registrados en la EMA-GF también pueden ser considerados dentro de un comportamiento normal (Fig. 6b). En la estación de Rothera el incremento anual total de la temperatura del aire fue de $2,7^{\circ} \mathrm{C}$ para el periodo desde 1978 a 2010, y para el verano de $0,8{ }^{\circ} \mathrm{C}$ (siendo sólo estadísticamente significativo con un nivel de confianza de un $95 \%$ para el valor anual). Este mismo incremento es estimado para el interior del GF. El promedio climatológico (19782010) anual de la temperatura derivado para la EMA-GF es de $-14,1 \pm 0,3{ }^{\circ} \mathrm{C}$, siendo en verano de $-7,4 \pm 0,1$ y en invierno de $-21,1 \pm 0,3{ }^{\circ} \mathrm{C}$ (note que los promedios obtenidos directamente de los datos de la EMA-GF para el 2008 son más cálidos que el promedio climatológico); por lo tanto, a pesar del probable aumento de la temperatura en el interior del GF, este está muy por debajo de la temperatura de congelamiento.

En promedio, la temperatura registrada en la EMA-GF fue $\sim 9.3^{\circ} \mathrm{C}$ más fría que la registrada en Rothera, lo que nos da un gradiente de temperatura vertical de $-0.88 \pm 0.27^{\circ} \mathrm{C} / 100 \mathrm{~m}$ para el periodo diciembre 2007 - octubre 2008. Este valor es similar al gradiente adiabático seco, cercano a -1 ${ }^{\circ} \mathrm{C} / 100$ m (Turner \& Pendlebury, 2004), pero mucho mayor que el gradiente altitudinal estimado por Reynolds (1981) para la península, que es de
$-0.57{ }^{\circ} \mathrm{C} / 100 \mathrm{~m}$. Esto nos revela más bien un ambiente seco en la parte alta del GF, en el lugar donde se emplazó la EMA. Durante el periodo de verano de 2008 , el gradiente de temperatura fue de alrededor de $-0.73 \pm 0.17^{\circ} \mathrm{C} / 100 \mathrm{~m}$, lo que indica un ambiente húmedo durante esa temporada.

El nivel de congelamiento en la atmósfera es la altitud a la cual la temperatura del aire es de $0^{\circ} \mathrm{C}$ y representa la altitud de la isoterma cero (ZIA en su sigla inglesa, Zero Isotherm Altitude). En la troposfera, bajo esta altitud la temperatura del aire es mayor a $0{ }^{\circ} \mathrm{C}$ y sobre ella es menor a 0 ${ }^{\circ} \mathrm{C}$. La ZIA fue calculada utilizando la temperatura diaria del aire superficial en la estación Rothera registrada a las 18 UTC, y el gradiente de temperatura de verano de $-0.64^{\circ} \mathrm{C} / 100 \mathrm{~m}$. Este gradiente se obtuvo con los datos de la temperatura del aire superficial de Rothera, la temperatura del aire a $850 \mathrm{hPa}$ y la altura geopotencial de 850 $\mathrm{hPa}$, utilizando datos del periodo 2004-2010 de las radiosondas de Rothera. Se optó por éste gradiente en desmedro del gradiente determinado para el verano del $2008\left(0.73 \pm 0.17^{\circ} \mathrm{C} / 100 \mathrm{~m}\right)$, ya que es mucho más apropiado para el cálculo aplicado a todo el periodo 1978-2010.

La Fig. 7 muestra las anomalías de la ZIA para todos los días con temperatura del aire superficial registrada a las $18 \mathrm{UTC}$ y que fue mayor a $0{ }^{\circ} \mathrm{C}$ en la estación Rothera ( $\mathrm{wd}$ ), durante la temporada de verano, (en adelante, $\mathrm{ZIA}_{\mathrm{wd}}$, altura de la isoterma cero para días cálidos). Las anomalías se calcularon en relación al promedio estival del periodo 19782010, y se optó por utilizar las observaciones a las 18 UTC debido a que es el momento cercano en que se puede registrar la temperatura máxima diaria del aire (Fig. 3), después de que se produce el máximo de la radiación solar entrante. Al utilizar solamente los días cálidos, permite analizar el comportamiento de la temperatura de la atmósfera más cercana a superficie afectando el GF (la parte baja cercana a la costa) y la WIS, cuando ésta está expuesta a temperatura ambiental sobre $0{ }^{\circ} \mathrm{C}$. El resto de los días, la $\mathrm{ZIA}_{\mathrm{wd}}$ estuvo ubicada al nivel de la superficie o por debajo de ella en la zona, ya que la temperatura del aire registrada en Rothera estuvo bajo los $0{ }^{\circ} \mathrm{C}$, es decir no hay probabilidad de descongelamiento. La temperatura del aire superficial en Rothera durante el verano (curva inferior en Fig. 6), revela un incremento de 0,25 

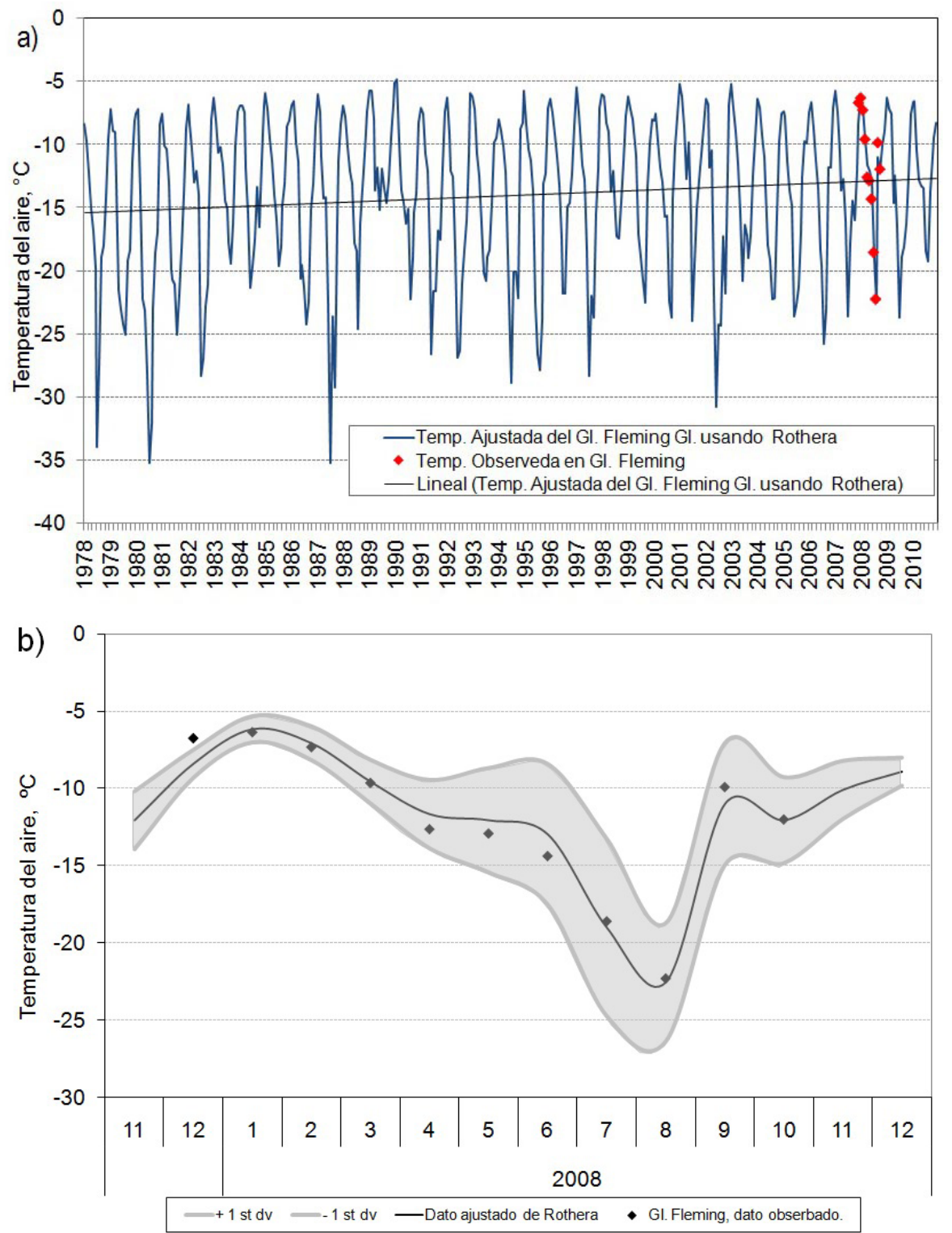

Fig. 6. a) Promedio mensual de la temperatura del aire registrada en la estación de Rothera pero ajustada a la locación de la EMA - GF utilizando la expresión $\mathrm{T}_{\mathrm{EMA}-\mathrm{FG}}=1.3114^{*} \mathrm{~T}_{\text {Rothera }}-8.3952$. b) Ajuste concurrente del promedio mensual con un \pm 1 de desviación estándar y los promedios mensuales observados en la EMA - GF.

${ }^{\circ} \mathrm{C} /$ década que es menor al aumento anual; de hecho, los datos muestran que este incremento ocurre durante la primera parte de los registros (1978-1990). En promedio, se contabilizaron $\sim 74$ \pm 9 (1 desviación estándar) días cálidos durante los veranos en Rothera, con una leve tendencia positiva durante el periodo 1978-2010, pero estadísticamente no significativa. Por su lado, la
ZIA $_{\text {wd }}$ en promedio se ubicó a 390 m.s.n.m a las 18 UTC. La tendencia lineal para la ZIA $_{\text {wd }}$ revela un incremento en altura estadísticamente significativo de $40 \mathrm{~m}$ para el periodo 1978-2010. En la Fig. 8 se muestra la ubicación aproximada de la ZIA para el año 1978 ( 360 m.s.n.m. línea roja) y para el 2010 ( 400 m.s.n.m. línea azul), utilizando la imagen dinámica de Google Earth. De acuerdo a 


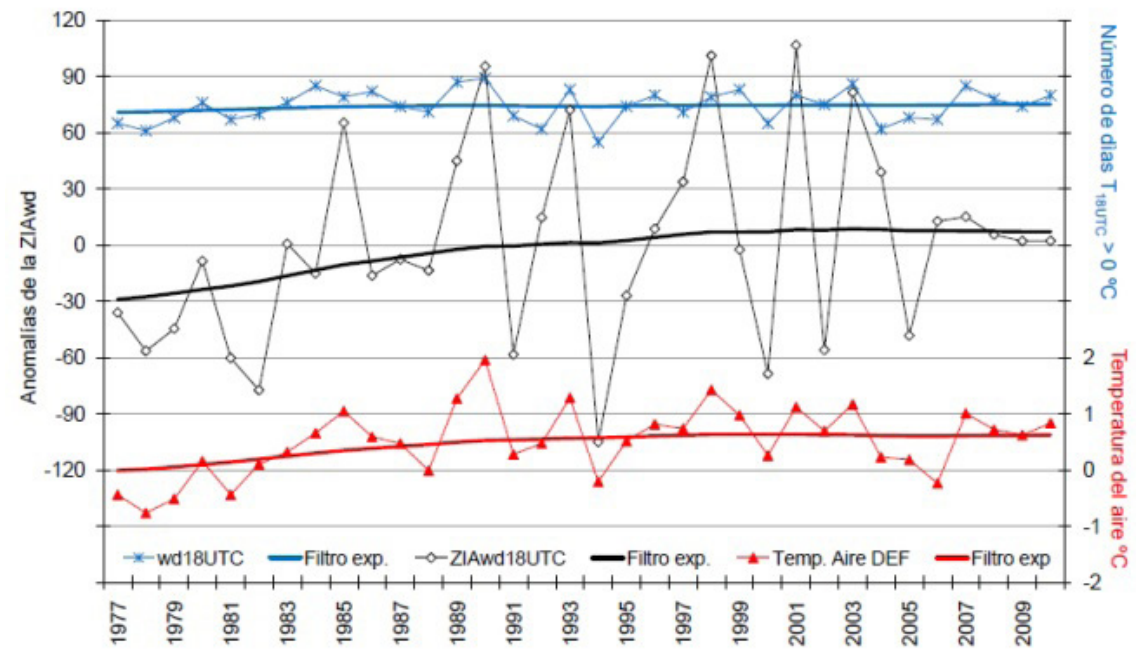

Fig. 7. Arriba en azul es el número de días con temperatura del aire superficial sobre los $0^{\circ} \mathrm{C}$. Al medio en negro son las anomalías de la altitud de la isoterma cero grado (ZIA $18 \mathrm{UTC}$ ). Abajo en rojo es la temperatura del aire promedio en verano en Rothera. Todas junto con la curva dada por el filtro exponencial.

la estimación realizada sobre la imagen el aumento de la altura de $40 \mathrm{~m}$ implica, para los días cálidos, un desplazamiento horizontal a nivel del suelo en el sector del glaciar Airy y Seller de 1030,6 $\pm 28,9$ $\mathrm{m}$ y en el sector del GF de $\sim 1123,5 \pm 70,0 \mathrm{~m}$.

\section{DISCUSIÓN Y CONCLUSIONES}

Como consecuencia del calentamiento regional observado en la PA, principalmente en su lado oeste, han ocurrido colapsos de las plataformas de hielo en décadas recientes. Su desintegración puede modificar la dinámica de los glaciares que anterior al colapso drenaban hacia plataformas de hielo sólidas y después directamente al mar. Uno de estos glaciares es el GF, tributario de la plataforma de hielo de Wordie. Caracterizar y analizar el comportamiento de la temperatura del aire en el parte superior del GF (cerca del límite norte del Plateau Dyer) ha sido descrita utilizando los datos

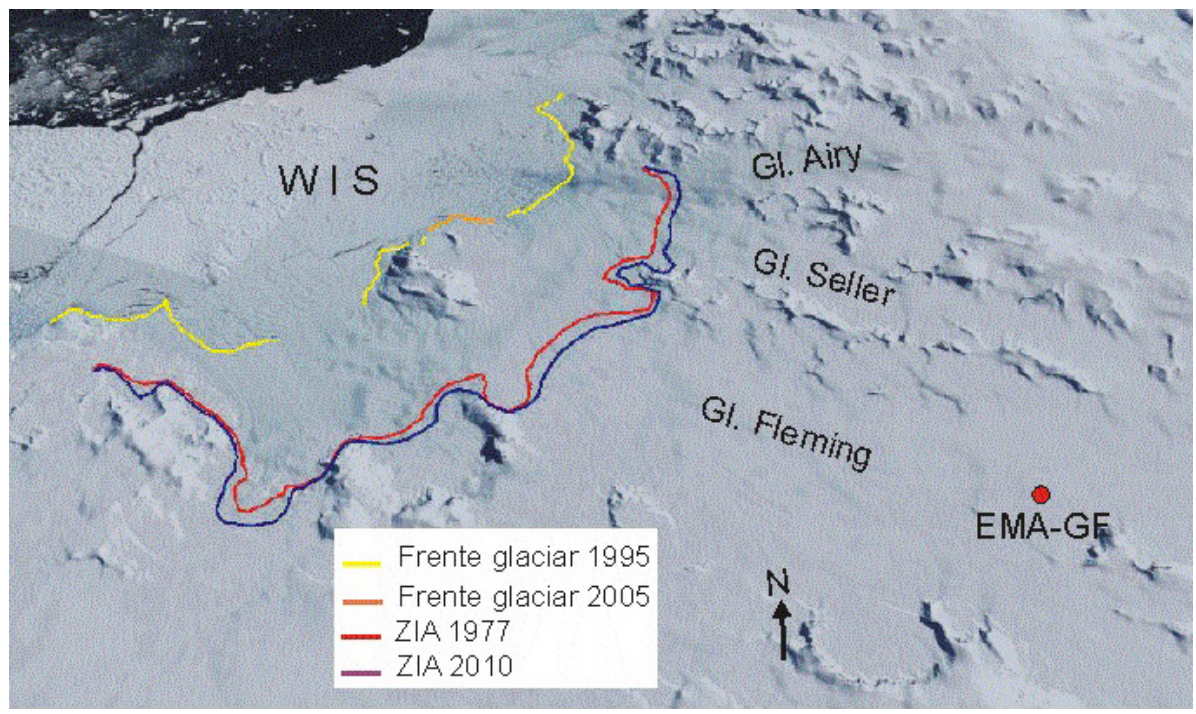

Fig. 8. Imagen Google Earth indicando la posición de la ZIA para 1978 (rojo) y 2010 (azul). 
recolectados in situ por una EMA instalada en noviembre de 2007, emplazada aproximadamente a unos $50 \mathrm{~km}$ al interior del frente del glaciar. Los datos registrados in situ, entre diciembre del 2007 y octubre del 2008, fueron comparados con datos meteorológicos adicionales registrados por estaciones meteorológicas cercanas, instaladas por el Programa de la Universidad de Wisconsin y datos de la estación inglesa Rothera, operada por el Servicio Antártico Británico.

La temperatura media mensual del aire registrada en la EMA-GF varió entre -6.3 \pm 0,1 y $-22,3 \pm 0,3{ }^{\circ} \mathrm{C}$ para el mes más cálido $y$ frío, respectivamente. Se encontraron importantes correlaciones estadísticas entre las variables registradas en la EMA-GF y las EMA circundantes en la zona. En particular, la alta correlación diaria (0.92) de la temperatura del aire que existe entre EMA-GF y Rothera, durante diciembre de 2007 y octubre de 2008, permite explorar la variabilidad de larga data que puede estar afectando el interior del GF y del Plateau Dyer. Según los datos de la estación Rothera ajustados a la EMA-GF, el año 2008 puede ser considerado un año dentro del rango normal, ya que los promedios mensuales están dentro de \pm 1 desviación estándar del promedio climatológico 1978-2010. Las temperaturas del aire de Rothera ajustadas al sitio de la EMA-GF, sugiere una tendencia de calentamiento anual de $0.69^{\circ} \mathrm{C} /$ década y una tendencia de calentamiento estival de $0.25^{\circ} \mathrm{C} /$ década, para el periodo $1978-$ 2010. El promedio anual, calculado con la media de verano e invierno de 2008 con los datos de la EMA-GF, fue de $-12,1 \pm 0,3{ }^{\circ} \mathrm{C}$; mientras que el promedio climatológico derivado de la serie de datos extendida ajustada a la altura y latitud de la EMA-GF fue de $-14.1 \pm 0,3{ }^{\circ} \mathrm{C}$. Estas cifras indican que el año 2008 fue más cálido que el promedio pero el incremento de la temperatura del aire en la región interior del GF, aún se mantiene bajo el punto de congelamiento.

Los resultados generales mostrados en la Fig. 7 no revelan una tendencia importante, para la temporada de verano durante los últimos 20 años alrededor de Rothera y probablemente en la parte baja del área del GF (incluyendo la plataforma de Wordie). La tendencia linear de invierno muestra un calentamiento estadísticamente importante de $1,2{ }^{\circ} \mathrm{C} /$ década para el periodo 1978-2010, utilizando las observaciones de las 12 UTC de Rothera (alrededor de la hora cuando es más probable que se produzca la temperatura mínima); las tendencia positiva más importante ocurre en las primeras dos décadas. Turner et al. (2005) señalan un calentamiento anual, invernal y estival de $1,01 \pm 1,42 ; \quad 0,36 \pm 0.57$ y $1,73 \pm 2,79$ ${ }^{\circ} \mathrm{C} /$ década, respectivamente; para el periodo 1978-2000, utilizando promedios diarios. Estos valores son comparables con la tendencia linear indicada en este estudio, pero menor debido a que el periodo de análisis es hasta el 2010, es decir, incluye la última década en donde el aumento de la temperatura global ha sido menor o decreciente en algunos lugares (Easterling \& Whener, 2009). De hecho, Carrasco (2012, 2013) encontró una tendencia positiva anual, invernal y estival de $0,69 \pm 1,31 ; 0,18 \pm 0,56$ y $1,09 \pm 2,87^{\circ} \mathrm{C} /$ década, respectivamente, en Rothera para el periodo 1978-2010.

Como el ciclo anual de la temperatura superficial del aire en la estación Rothera indica que se superan los $0{ }^{\circ} \mathrm{C}$ sólo durante el verano; para el análisis de posibles cambios de la altitud de la isoterma cero (ZIA), se utilizaron sólo los datos diarios cuando la temperatura superficial del aire registrada a las $18 \mathrm{UTC}$ fue $>0{ }^{\circ} \mathrm{C}\left(\mathrm{ZIA}_{\mathrm{wd}}\right)$. La altitud media de la ZIA $_{w d}$ durante el verano, en esta localidad y para la región de estudio fue de 390 m.s.n.m. Esta altura se alcanza a una distancia de alrededor de $15-17 \mathrm{~km}$ al interior del frente estimado del glaciar (Fig. 8). Por otra parte, es durante los veranos que en el área de interior del GF pueden ocurrir eventos con temperatura superior a $-2,5^{\circ} \mathrm{C}$; es decir, sobre el umbral de descongelamiento para PA según Zwally \& Fiegles (1994). Para el verano de 2008 se registraron 125 horas $>-2,5^{\circ} \mathrm{C}$, distribuidos en 29 días. Sin embargo, estos eventos son de duración menor a un día y de pocas horas de duración, por lo tanto, la ocurrencia de ablación sobre los 390 m.s.n.m. durante el verano es muy poco probable, revelando que la mayor parte del GF y sus áreas circundantes están bajo condiciones de congelamiento durante todo el año, y muy probablemente sin cambios significativos durante el periodo 1978-2010. 


\section{AGRADECIMIENTOS}

Este trabajo se desarrolló con financiamiento del Proyecto Anillo Conicyt-Bicentenario ARTG022006 "Stability and recent behaviour of glaciers in Antarctic Peninsula the interactions with ice shelves".

\section{LITERATURA CITADA}

Carrasco, J.F. (2012). Red de estaciones de observación atmosférica en la Antártica, una colaboración internacional para la investigación del cambio climático. Anales Instituto Patagonia, 40(1), 57-63.

Carrasco, J.F. (2013). Decadal changes in the near-surface air temperature in the western side of the Antarctic Peninsula. Atmosphere and Climate Sciences, 3, 275-281.

Cook, A.J. \& Vaughan, D.G. (2010). Overview of areal changes of the ice shelves on the Antarctic Peninsula over the past 50 years. The Cryosphere, 4, 77-98, doi:10.5194/ tc-4-77-2010.

Din, Q., Batisti, D.S., \& Kuttel, M. (2011). Winter warming in West Antarctica caused by central tropical Pacific warming. Nature Geoscience, 4, 398-403, doi: 10.1038/ ngeo1129.

Doake, C.S.M. (1975). Bottom sliding of a glacier measured from the surface. Nature, 257, 780-782.

Easterling, D. R., \& Whener, M. F. (2009). Is the climate warming or cooling? Geophysical Research Letters, 36, L08706, doi: 10.1029/2009GL037810.

Hock, R., De Woul, M., Radic, V., \& Dyurgerov, M. (2009). Mountain glaciers and ice caps around Antarctica make a large sea-level rise contribution. Geophysical Research Letters, 36, L07501, doi:10.1029/2008GL0307020.

Marshall, G.J., Orr, A., van Lipzig, N.P.M., \& King, J.C. (2006). The impact of a changing Southern Hemisphere Annular Mode on Antarctic Peninsula summer temperatures. Journal of Climate, 19, 5388-5404.

Morris, E. M., \& Vaughan, D. G. (2003). 'Spatial and temporal variation of surface temperature on the Antarctic Peninsula and the limit of viability of ice shelves', In Domack, E., Leventer, A., Burnett, A., Bindschadler, R., Convey, P., and Kirby, M. (eds.), Antarctic Peninsula Climate Variability: Historical and Paleoenvironmental Perspectives. Antarctic Research Series, 79, AGU, Washington, DC. pp. 61-68.

Reynolds, J.M. (1981). The distribution of mean annual temperatures in the Antarctic Peninsula. British Antarctic Survey Bulletin, 54, 123-133.

Rignot, E., Casassa, G., Gogineni, P., Krabill, W., Rivera, A., \& Thomas, R. (2004). Accelerated ice discharge from the Antarctic Peninsula following the collapse of Larsen B ice shelf. Geophysical Research Letters, 31(18), L18401. doi:10.1029/2004GL020697.

Rignot, E., Casassa, G. , Gogineni, S., Kanagaratnam, P., Krabill, W., Pritchard, H. , Rivera, A., Thomas, R., Turner. J., \& Vaughan, D. (2005). Recent ice loss from the Fleming and other glaciers, Wordie Bay, West Antarctic Peninsula. Geophysical Research Letters, 32, L07502, doi:10.1029/2004GL021947.

Rosenblüth, B., Fuenzalida, H.A., \& Aceituno, P. (1997). Recent temperatures variations in southern South America. International Journal of Climatology, 17, 67-85.

Schwerdtfeger, W. (1984). Weather and Climate of the Antarctic. Elsevier, New York.

Smith, R.C., \& Stammerjohn, S.E. (1996). Surface air temperature variations in the western Antarctic Peninsula region. Antarctic Research Series, 70, 105-121.

Turner, J., Lachlan-Cope, T.A., Colwell, S., Marshall, G.J., \& Connolley, W.M. (2006). Significant warming of the Antarctic winter troposphere. Science, 311, 1914-1917.

Turner, J. \& Pendlebury, S. (2004). The International Antarctic weather forecast handbook. Publish by British Antarctic Survey.

Turner, J., Comiso, J.C., Marshall, G.J., LachnCope, T.A., Cartelon, M.A., Jones, P.D., Lagun, V., Reid, P.A., \& lagovkina, S. (2005). Antarctic Climate change during the last 50 years. International Journal of Climatology, 25(8), 279-294. doi: 10.1002/joc. 1130. 
Vaughan, D.G. (2006). Recent trends in melting conditions on the Antarctic Peninsula and their implications for ice-sheet mass balance and sea level. Artic, Antarctic, and Alpine Research, 38(1), 147-152.

Vaughan, D.G., Bamber, J.L., Giovinetto, M., Russell, J., \& Cooper A.P. (1999). Reassessment of net surface mass balance in Antartica. Journal of climate, 12, 933-946. Vaughan, D.G., \& Doake C.S.M. (1996). Recent atmospheric warming and retreat of ice shelves on the Antarctic Peninsula. Nature, 379(6563), 328-331.
Vaughan, D.G., Marshall, G.J., Connolley, W.M., King, J.C., \& Mulvaney, R. (2001). Climate change: devil in the detail. Science, 293 (5536), 1777-1779.

Wendt, J., Rivera, A., Bown, F., Wendt, A., Zamora, R., Casassa, G., \& Bravo, C. (2010). Recent ice elevation changes of Fleming glacier in response to the removal of Wordie ice shelf, Antarctic Peninsula. Annals of Glaciology. 51(55), 97-102.

Zwally, J.J., \& Fiegles, S. (1994). Extent and duration of Antarctic surface Melting. Journal of Glaciology, 40(136), 463-476. 
